\title{
Association of Trait Anxiety and Social Desirability with White Blood Cell Counts
}

\author{
Riichiro Ishida* and Masahiko Okada
}

\author{
Division of Clinical Preventive Medicine, Niigata Graduate School of Medical and Dental Sciences, Niigata University, \\ Japan
}

\begin{abstract}
The present study aimed to examine the association of trait anxiety and social desirability (SOD) with granulocyte (GRA) and lymphocyte (LYM) counts in healthy adults. Subjects were 23 ( 9 males and 14 females) eligible adults. Trait anxiety and SOD were assessed using the Anxiety and Lie Scores of the Manifest Anxiety Scale (MAS), respectively. White blood cells were counted by flow cytometry. Data were analyzed by logistic regression analyses. The results showed that the odds ratio (OR) of MAS score was significant for prediction of GRA count (OR: 1.399, $\mathrm{p}=0.032)$ and also for neutrophil (NEU) count (OR: 1.381, $\mathrm{p}=0.040$ ). The OR of Lie Scale scores was significant for LYM count (OR: $0.165, \mathrm{p}=0.036)$ and also for GRA count (OR: $1.879, \mathrm{p}=0.048)$. The present study suggests that healthy subjects with higher trait anxiety have a higher GRA count.
\end{abstract}

Keywords: Trait anxiety, social desirability, granulocyte count, lymphocyte count.

\section{INTRODUCTION}

Anxiety is usually accompanied by increased sympathetic nervous system activity $[1,2]$. This psychological state consists of state anxiety and trait anxiety, with the former occurring in response to specific stresses, and the latter occurring as a personal characteristic. Both types of anxiety are strongly correlated with one another. Trait anxiety can be assessed by Manifest Anxiety Scale (MAS), which comprises the Lie Scale to assess the reliability of an individual's responses [3, 4]. The Lie Scale is also used to evaluate an individual's attitudes related to showing off. Social desirability (SOD) is one of the attitudes that reflect perceived social norms and values $[5,6]$ and can easily cause either state and/or trait anxiety under stressful situations [7].

High anxiety is a possible risk factor for various diseases $[8,9]$, including coronary heart disease, in which an imbalance of the autonomic nervous system plays an important role [10]. Recently, Abo et al. reported that the autonomic nervous system regulates the production of both lymphocytes and granulocytes. They found that high sympathetic activity increased granulocyte (GRA) count, and high parasympathetic activity increased lymphocyte (LYM) count $[11,12]$. GRAs are a type of white blood cell, and include neutrophils (NEUs), eosinophils (EOSs) and basophils (BASs). GRAs, particularly NEUs, ingest bacteria by phagocytosis and then release enzymes to destroy them. LYMs are also a type of white blood cell, and they play an important role in defending the host from both tumors and

*Address correspondence to this author at the Division of Clinical Preventive Medicine, Niigata Graduate School of Medical and Dental Sciences, Niigata University, Asahimachi 1, Chuo-ku, Niigata 951-8510, Japan; Tel: +81-25227-2336; Fax: +81-25-223-0996; E-mail: ishida@med.niigata-u.ac.jp virally infected cells. Increases in GRA count are usually observed in suppurative diseases, while increases in LYM count are seen in viral infections or immunological diseases.

The present study aimed to examine the association of trait anxiety and SOD with GRA and LYM counts in healthy adults using the MAS and Lie Scale.

\section{METHODS}

\section{Subjects}

In the present study, we recruited 25 adults (11 males and 14 females). Two male subjects were excluded: one subject (age, 42 years) could not understand the questions in the Japanese version of Manifest Anxiety Scale (MAS), as Japanese was not his native language; the other (age, 43 years) reported that he was ill on the day of blood testing. Thus, subjects were 23 (9 males and 14 females) eligible adults. The ethics committee of the Niigata University School of Medicine approved the study and all subjects provided written informed consent.

\section{Measurement of Trait Anxiety and SOD}

We used a Japanese version of MAS that consists of 50 items for assessing trait anxiety and 15 items for the Lie Scale [3]. The Lie Scale contains several items to assess the status recognized as desirable, but impracticable as follows: 'I do not dislike anyone' or 'I never laugh at another person's bad joke'. Subjects responded using a 2-point Likert scale ('yes' or 'no'). Trait anxiety score theoretically ranges from 0 to 50 points, with higher scores indicating higher levels of trait anxiety. Scores for Lie Scale theoretically range from 0 to 15 points, with higher scores indicating stronger levels of SOD. 


\section{Blood Testing}

Twenty milliliters of blood were taken from the antecubital vein of the forearm [13, 14]. White blood cells were counted by flow cytometry. As white blood cell counts are significantly correlated with food, time, posture and weather conditions, particularly atmospheric pressure (ATM) [15], blood was taken in the morning between 10:00 and 11:00 a.m. Participants were instructed not to consume any breakfast, alcohol, coffee or tea before testing. An air conditioner was used to maintain room temperature and humidity at constant levels. Blood testing was performed on a subject-by-subject basis within a week following completion of the MAS.

\section{Statistical Analyses}

Data are presented as means \pm standard deviation. Pearson's correlation analysis was performed to determine univariate associations among six parameters: gender, age, BMI, MAS score, Lie score, and ATM. For multivariate analyses, two logistic regression models were obtained. For dependent variables, 1 and 2 were assigned to high and low counts for each type of white blood cell, respectively. In addition, 1 and 2 were assigned to male and female, respectively. Results were considered statistically significant when $\mathrm{p}<0.05$. The software package SPSS (SPSS Japan Inc., Tokyo, Japan) was used for statistical analysis.

\section{RESULTS}

Mean age of the subjects was $23.04 \pm 8.29$ years, with a range of 18 to 50 years (Table 1). The mean ATM on the days of blood sampling was $1013.05 \pm 3.37 \mathrm{hPa}$.

Table 1. Baseline Characteristics of Subjects and Weather Conditions $(\mathbf{N}=\mathbf{2 3})$

\begin{tabular}{|c|c|c|c|}
\hline & Mean \pm SD & MIN & MAX \\
\hline \multicolumn{4}{|c|}{ Demographic Parameters } \\
\hline Age (years) & $23.04 \pm 8.29$ & 18 & 50 \\
\hline Height $(\mathrm{cm})$ & $164.22 \pm 8.29$ & 149 & 185 \\
\hline Weight (kg) & $56.48 \pm 8.91$ & 40 & 73 \\
\hline $\operatorname{BMI}\left(\mathrm{kg} / \mathrm{m}^{2}\right)$ & $20.85 \pm 2.22$ & 16.44 & 24.61 \\
\hline \multicolumn{4}{|l|}{ Weather Parameter } \\
\hline $\operatorname{ATM}(\mathrm{hPa})$ & $1013.05 \pm 3.36$ & 1007.30 & 1020.00 \\
\hline \multicolumn{4}{|c|}{ Psychological Parameters } \\
\hline MAS score (points) & $17.74 \pm 4.96$ & 7 & 26 \\
\hline Lie score (points) & $4.43 \pm 1.97$ & 0 & 8 \\
\hline
\end{tabular}

SD: standard deviation, MIN: minimum, MAX: maximum, BMI: body mass index, ATM: atmospheric pressure, and MAS: manifest anxiety scale.

On univariate analyses, the following significant differences were observed: LYM count between the high and

\section{$($ count $/ \mu \mathrm{l})$}

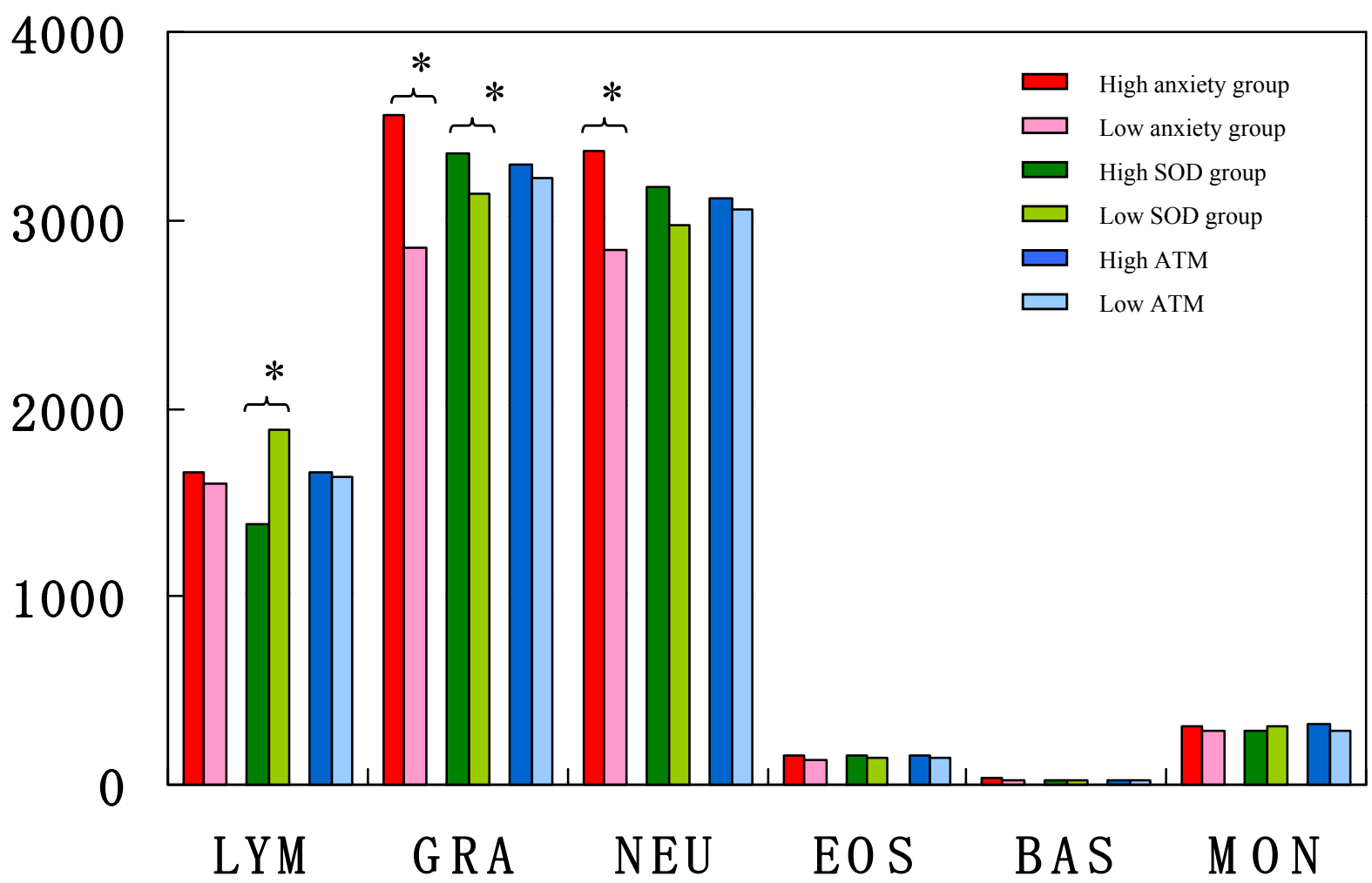

Fig. (1). Mean count of lymphocytes (LYM), granulocytes (GRA) and its components, i.e., neutrophils (NEU), eosinophils (EOS) and basophils (BAS), and monocytes (MON). SOD: social desirability, ATM: atmospheric pressure. ${ }^{*}: \mathrm{p}<0.05$. 
Table 2. Adjusted Odds Ratios of MAS Score, Lie Scale Score and ATM for Predicting White Blood Cell Counts

\begin{tabular}{|c|c|c|c|c|c|c|c|c|c|c|c|c|}
\hline & \multicolumn{2}{|c|}{ Lymphocyte } & \multicolumn{2}{|c|}{ Granulocyte } & \multicolumn{2}{|c|}{ Neutrophil } & \multicolumn{2}{|c|}{ Eosinophil } & \multicolumn{2}{|c|}{ Basophil } & \multicolumn{2}{|c|}{ Monocyte } \\
\hline & OR & $\mathbf{P}$ & OR & $\mathbf{P}$ & OR & $\mathbf{P}$ & OR & $\mathbf{P}$ & OR & $\mathbf{P}$ & Odds Ratio & $\mathbf{P}$ \\
\hline MAS score & 0.860 & 0.381 & 1.399 & 0.032 & 1.381 & 0.040 & 1.072 & 0.505 & 0.869 & 0.193 & 0.922 & 0.410 \\
\hline Lie score & 0.165 & 0.036 & 1.879 & 0.048 & 1.675 & 0.083 & 0.954 & 0.851 & 0.621 & 0.110 & 0.789 & 0.350 \\
\hline ATM & 1.309 & 0.180 & 0.813 & 0.219 & 0.824 & 0.245 & 1.091 & 0.538 & 1.203 & 0.222 & 1.181 & 0.249 \\
\hline
\end{tabular}

low SOD groups; GRA count between high and low anxiety groups and also between high and low SOD groups; and NUE count between high and low anxiety groups (Fig. 1). No significant correlations were observed among the six parameters (data not shown). In addition, no significant correlations were observed between the MAS scores and Lie scores. Logistic regression analyses showed that odds ratio (OR) of MAS score was significant for the prediction of GRA count (OR: 1.399, $p=0.032)$ and also for NEU count (OR: 1.381, $\mathrm{p}=0.040$ ) (Table 2). OR of the Lie Scale score was significant for LYM count (OR: $0.165, p=0.036)$ and also for GRA count (OR: 1.879, $\mathrm{p}=0.048$ ). However, ORs for gender, age, BMI and ATM were not significant for any LEU parameters.

\section{DISCUSSION}

We examined the associations of trait anxiety and SOD with white blood cell parameters and found that anxiety level was positively associated with GRA and NEU counts, respectively, but not with LYM count. SOD levels were also associated positively with GRA count and negatively with LYM count. Gender, age, BMI, ATM, MAS score and Lie Scale score were not significantly associated with any of the white blood cell parameters. The most significant finding in the present study was that trait anxiety was positively associated with GRA and NEU counts. This result is not necessarily compatible with the existing data that trait anxiety is not obviously associated with GRA count $[14,16-$ $18]$.

High GRA count represents suppurative diseases [17, 18], and high LYM count represents viral infection or immunological diseases [11, 14, 16-19]. Therefore, the results show that both trait anxiety and SOD are possible risk factors in various diseases. Numerous epidemiological studies have shown that persons with type A behavior patterns exhibiting aggressive and competitive characteristics are accompanied by high sympathetic nervous activity and in turn tend to have coronary heart disease $[1,20]$. Other studies have shown that higher SOD stimulates the sympathetic nervous system under high-stress conditions [21]. Therefore, it may be concluded that trait anxiety and SOD are possible risk factors for coronary heart disease [3, 7].

Limitations of the present study include the small number of subjects. The results must therefore be confirmed in a future study with more subjects. Autonomic nervous system function is readily influenced by several factors. Although, in the present study, confounding factors such as BMI and
ATM were statistically controlled, and blood sampling was performed at a fixed time, temperature, and humidity, other factors such as alcohol intake, habitual smoking, and exercise should be considered in a future study.

The results of this study suggest that healthy subjects with higher trait anxiety have higher GRA count.

\section{REFERENCES}

[1] Waldinger RJ. Psychiatry. 2nd ed. Washington, DC: American Psychiatric Press 1990.

[2] Schmidt RA. Motor control and learning. Champaign: Human Kinetics Publishers 1988.

[3] Taylor JA, Abe M, Takaishi N. MAS (The Japanese version manual). Kyoto: Sankyo-bo 1943. (in Japanese)

[4] Tayler JA. A personality scale of manifest anxiety. J Abnorm Soc Psychol 1953; 48: 285-91.

[5] Smith EE, Nolen-Hoeksema, Fredricson BL, Loftus GR. Atkinson \& Hilgard's Introduction to psychology. 4 th ed. Belmont, CA: Thomson 2003.

[6] Colman AM. A dictionary of psychology. 3 rd ed. New York, Oxford University Press 2001.

[7] Motl RW, McAuley E, DiStefano C. Is social desirability associated with self-reported physical activity? Pre Med 2005; 40: 735-9.

[8] Dichter GS. Anhedonia in unipolar major depressive disorder: a review. Open Psychiatr J 2010; 4: 1-9.

[9] Voorhees BWV. Adolescents in primary care with sub-threshold depressed mood screened for participation in a depression prevention study: co-morbidity and factors associated with depressive symptoms. Open Psychiatr J 2010; 4: 10-8.

[10] Hemingway H, Shipley M, Brunner E, et al. Does autonomic function link social position to coronary risk? Circulation 2005; 111: 3071-7.

[11] Guyton AC. Textbook of medical physiology. 8th ed. London: W.B. Saunders Company 1991.

[12] Abo T, Kawamura T. Immunomodulation by the autonomic nervous system: therapeutic approach for cancer, collagen diseases, and inflammatory bowel diseases. Ther Apher 2002; 6(5): 348-57.

[13] Zhang Y, Hansen JK, Xiang L, et al. A flow cytometry method to quantitative internalized immunotoxins shows that taxol synergistically increases cellular immunotoxins uptake. Cancer Res 2010; 70 (3): 1082-9.

[14] Di Donato A, Di Giampaolo L, Real M, et al. Effect of occupational stress and anxiety on natural killer lymphocyte activity of men and women employed in a university. Int $\mathrm{J}$ Immunopathol Pharmacol 2006; 19(4): 79-84.

[15] Fukuda M, Moroda T, Toyabe S, et al. Granulocytosis induced by increasing sympathetic nerve activity contributes to the incidence of acute appendicitis. Biomed Res 1996; 17(2): 171-81.

[16] Pekiner FN, Demirel GY, Gümrü B, Özbayrak S. Serum cytokine and $\mathrm{T}$ regulatory cell in patients with burning mouth syndrome. $\mathrm{J}$ Oral Pathol Med 2008; 37: 528-34.

[17] Fredrikson M, Füsrst CJ, Lekander M, Rotstein S, Blomgren H. Trait anxiety and anticipatory immune reactions in women receiving adjuvant chemotherapy for breast cancer. Brain Behav Immun 1993; 7(1): 79-90.

[18] Keresztes M, Rudisch T, Tajti J, Ocsovszki I, Gardi J. Granulocyte activation in humans is modulated by psychological stress and relaxation. Stress 2007; 10(3): 271-81. 
[19] Goldsby RA, Kindt TJ, Osborne BA, Kudy J. Immunology. 5 th ed. New York, Freeman and Company 2003.

[20] Bandura A. Social learning theory. Upper Saddle River: Prentice Hall 1977.
[21] Miller SB. Cardiovascular reactivity in anger-defensive individuals: the influence of task demand. Psychosom Med 1993; 55: 78-85.

Received: March 8, 2010

Revised: October 1, 2010

Accepted: December 24, 2010

(C) Ishida and Okada; Licensee Bentham Open.

This is an open access article licensed under the terms of the Creative Commons Attribution Non-Commercial License (http://creativecommons.org/licenses/by-nc/

$3.0 /$ ) which permits unrestricted, non-commercial use, distribution and reproduction in any medium, provided the work is properly cited 